\title{
Assessment of coronary artery disease using 3.0 T magnetic resonance coronary angiography: a national multicenter trial
}

Qi Yang ${ }^{1 *}$, Kuncheng Li ${ }^{1}$, Bin Sun ${ }^{2}$, Hong Yun ${ }^{3}$, Lijun Tang ${ }^{4}$, Shurong $\mathrm{Li}^{6}$, Zhenbin Cao ${ }^{8}$, Junling X ${ }^{5}$, Mengqi Wei ${ }^{7}$, Lixin $\operatorname{Jin}^{9}$

From 16th Annual SCMR Scientific Sessions

San Francisco, CA, USA. 31 January - 3 February 2013

\section{Background}

3.0T contrast enhanced whole-heart coronary magnetic resonance angiography (MRA) is a promising method for noninvasive, radiation-free detection and exclusion of obstructive coronary artery disease (CAD); however, the accuracy of this approach has not been determined in a multicenter trial.

\section{Methods}

An ECG-triggered, navigator-gated, inversion-recovery prepared, segmented gradient-echo sequence was used for image acquisition in 272 patients with suspected CAD at 8 hospitals. The accuracy of coronary MRA for detecting a $50 \%$ diameter reduction was determined using X-ray coronary angiography as the reference method. Using an

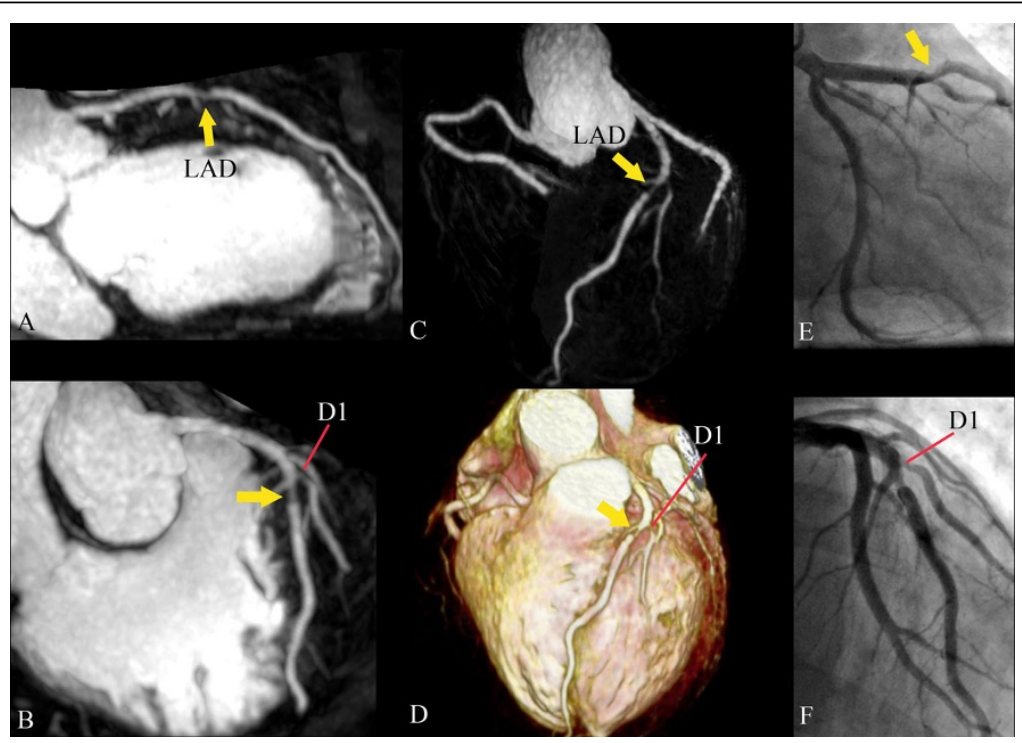

Figure 1 Curved planar reconstruction (CPR) image (A), Sliding thin slab maximum intensity projection (MIP) image (B), MIP image of coronary tree (C), and volume-rendered image (D) detect coronary artery stenoses in the LAD (arrow) and first diagonal branch (arrowhead). Good agreement is observed between coronary MRA and X-ray coronary angiography.

$\overline{1}$ Radiology Department, Xuanwu Hospital, Capital Medical University, Beijing,

China

Full list of author information is available at the end of the article 
intention-to-diagnose approach, all coronary arteries were included for the evaluation regardless of the image quality of coronary MRA to avoid overestimation of the diagnostic accuracy. Clinical Trial Registration-URL: http:// clinicaltrials.gov. Unique identifier: NCT01024478.

\section{Results}

Acquisition of coronary MRA was successfully completed in 235 of $272(86 \%)$ patients with average imaging time of $9.5 \pm 1.6$ minutes. The areas under the receiver-operator characteristic curve from MRA images according to vessel- and patient-based analyses were 0.90 (95\% confidence interval [CI]: 0.88 to 0.95 ) and 0.88 (95\% CI: 0.83 to 0.93 ), respectively. The sensitivity and specificity of MRA on per-patient basis were $91 \%$ and $80 \%$, respectively.

\section{Conclusions}

Among patients who were scheduled to obtain conventional $\mathrm{x}$-ray coronary angiography, we found that coronary MRA at 3.0T demonstrates high accuracy for detection of significant coronary artery stenosis. It warrants greater consideration as a suitable noninvasive method to exclude obstructive CAD.

\section{Funding}

National Basic Research Program 973 (grant no. 2010CB732600) from Ministry of Science and Technology, China; National Natural Science Foundation of China, grant number 30900355; National Institute of Health, grants numbers NIBIB EB002623 and NHLBI HL38698.

\footnotetext{
Author details

${ }^{1}$ Radiology Department, Xuanwu Hospital, Capital Medical University, Beijing, China. ${ }^{2}$ Radiology Department, Fujian Union Hospital, Fujian Medical University, Fuzhou, China. ${ }^{3}$ Radiology Department, Zhongshan hospital, Fudan University, Shanghai, China. ${ }^{4}$ Radiology Department, Jiangsu Province Hospital, Nanjing Medical University, Nanjing, China. ${ }^{5}$ Radiology Department, Henan Provincial Hospital, Zhengzhou, China. ${ }^{6}$ Radiology Department, First Affiliated Hospital, Sun Yat-Sen University, Guangzhou, China. ${ }^{7}$ Radiology Department, Xijing Hospital, Fourth Military Medical University, Xian, China. ${ }^{8}$ Radiology Department, Wuhan Union Hospital, Huazhong University of Science, Wuhan, China. ${ }^{9}$ Siemens Healthcare, MR Collaboration NE Asia, Shanghai, China.
}

Published: 30 January 2013

doi:10.1186/1532-429X-15-S1-E5

Cite this article as: Yang et al:: Assessment of coronary artery disease using 3.0 T magnetic resonance coronary angiography: a national multicenter trial. Journal of Cardiovascular Magnetic Resonance 2013 15(Suppl 1):E5.

\section{Submit your next manuscript to BioMed Central} and take full advantage of:

- Convenient online submission

- Thorough peer review

- No space constraints or color figure charges

- Immediate publication on acceptance

- Inclusion in PubMed, CAS, Scopus and Google Scholar

- Research which is freely available for redistribution

Submit your manuscript at www.biomedcentral.com/submit
C Biomed Central 